\title{
First Report of Preimplantation Genetic Diagnosis for Steroid-Resistant Nephrotic Syndrome
}

\author{
Faravareh Khordadpoor Deilamani (iD) ${ }^{1}$ and Mohammad Taghi Akbari (iD) 1, \\ ${ }^{1}$ Tehran Medical Genetics laboratory, Tehran, Iran \\ ‘Corresponding author: Tehran Medical Genetics laboratory, Tehran, Iran. Email: akbarimt@gmail.com
}

Received 2020 September 05; Accepted 2020 October 12.

\begin{abstract}
Background: Steroid-resistant nephrotic syndrome is a genetic disease with autosomal recessive inheritance pattern and symptoms such as proteinuria and hypoalbuminemia and rapid progress of kidney disease. Preimplantation genetic diagnosis is an option for couples who are at risk of affected pregnancy to have a healthy child.

objectives: This study aimed to develop a new PGD test for a couple who are heterozygous for a mutation in NPHS2 gene and have a son affected to steroid-resistant nephrotic syndrome.

Methods: Variant detection by cycle sequencing and Multiplex fluorescent PCR for identification of flanking STR markers were used to investigate the status of the embryos.

Results: Three out of six embryos were transferable from which one was transferred and resulted in the birth of a healthy boy. Conclusions: We recommend increasing the number of the STR markers to two at the downstream of the NPHS2 gene especially in cases that direct mutation analysis such as cycle sequencing is not applied.
\end{abstract}

Keywords: Preimplantation Genetic Diagnosis, Nephrotic Syndrome Type 2, Steroid-Resistant Nephrotic Syndrome

\section{Background}

Steroid-resistant nephrotic syndrome is an autosomal recessive kidney disorder with the symptoms of early childhood onset of proteinuria, edema, hypoalbuminemia, hyperlipidemia, rapid progression to end-stage renal disease and focal segmental glomerulosclerosis. This disease is caused by mutations in NPHS2 gene which is located on chromosome 1q25.2. Podocin is an integral membrane protein which is encoded by NPHS2 gene in the podocytes of kidney glomeruli. It has an important role in the function of the glomerular filtration barrier (1-3).

Due to absence of treatment for most genetic diseases, prevention of these disorders is still crucial. Prenatal diagnosis can identify a genetic disorder during the pregnancy for couples with a risk of having genetic disorders. However in some cases, the termination of one or more pregnancy is unavoidable. Preimplantation genetic diagnosis is another option in which the healthy embryos are selected before implantation and abolishes the need for pregnancy termination. Three decades has passed from the first time that PGD was applied for human (4-6). So far, PGD has been applied for many genetic disorders such as alpha and beta thalassaemia, Phenylketonuria (PKU), Dystonia, Neurofibromatosis and etc. (7-12).

\section{Objectives}

Here we report the first use of PGD for steroid-resistant nephrotic syndrome in a couple with an affected child. This screening resulted in the birth of a healthy boy.

\section{Methods}

\subsection{Patients}

A couple (35 year old female and 43 year old male) with consanguineous marriage and having a five year old son affected with steroid-resistant nephrotic syndrome were referred to the PGD center of Tehran Medical Genetics Laboratory. In the same laboratory the homozygous mutation of c.156delG (p.Thr53Profs*46) in NPHS2 gene had been previously found in the affected son by whole exome sequencing (WES). So the risk of producing another affected child for this family was $25 \%$ in each pregnancy. 
After PGD counseling, the informed consent was obtained from the parents. The study was approved by the local ethics committee.

\subsection{In Vitro Fertilization}

The IVF procedure and embryo biopsy was performed in an IVF center. Six embryos were produced.

After washing in PBS, the biopsied cells were transferred to our PGD center each in a tube containing 2-2.5 $\mu \mathrm{l}$ of PBS.

\subsection{Whole Genome Amplification}

Whole genome amplification (WGA) was performed on each blastomere and positive and negative control samples using the Picoplex WGA kit (Rubicon Genomics) according to the manufacturer's protocol. Briefly this WGA protocol includes 3 steps of lysis/DNA extarction, preamplification and amplification. Success of the WGA was assessed by running $5 \mu \mathrm{l}$ of the products on $1.5 \%$ agarose gel. The rest of the products were purified by QIAquick PCR purification kit from QIAGEN.

\subsection{Cycle sequencing and Linkage Analysis}

Two different tests including Cycle sequencing of exon 1 of NPHS2 gene and STR based linkage analysis were used to study the transmission of c.156delG (p.Thr53Profs*46) mutation in NPHS2 gene to the embryos.

Three upstream (D1S2883, D1S3759 and D1S215) and one downstream (D1S3760) dinucleotide STR markers were included in linkage analysis. The distance between the farthest STR markers from each other was less than $800 \mathrm{~Kb}$ (Figure 1) in order to minimize the probability of the recombination. Primer design was carried out using primer blast tool (https://www.ncbi.nlm.nih.gov/tools/primerblast/), GeneRunner software and SNPcheck website (https://genetools.org/SNPCheck/snpcheck.htm).

The size of the PCR products was considered to be less than 270bp in order to decrease the probability of allele and locus drop out (Table 1). Forward primers of the STR markers were fluorescently labeled with FAM. The markers were amplified by multiplex PCR and the fragments were separated on 3130 Genetic Analyzer (Foster City, CA, USA) using GS 500 Liz size standard and the data were analyzed by GeneMarker software (State College, PA, USA). Sequencing results were analyzed using Chromas software and blastn.

\section{Results}

Cycle sequencing confirmed the whole exome sequencing result and the c.156delG (p.Thr53Profs*46) mutation was detected in exon 1 of NPHS2 gene (Figure 2). Also the segregation of the mutation was confirmed in the family.

The haplotype of the STR markers which were linked to the mutated NPHS2 allele was inferred by investigating the parents and their affected son.

At the PGD day, the biopsied cells from six embryos were received from the IVF center. Whole genome amplification for two embryos (embryos 3 and 6) was not successful and could not be processed in later stages. For the rest of the embryos STR-based linkage analysis and cycle sequencing were performed (Figures 3 and 4).

Embryos 1, 4 and 5 were transferable. However, since only one embryo was decided to be transferred based on the discretion of the physician, our priority was the transfer of embryo 4 which was confirmed to inherit at least one normal allele by both sequencing and STR analysis and there was the chance that the second allele was normal too. So the embryo 4 was implanted and the pregnancy occurred successfully.

PND was done at 12th week of gestation and confirmed the conception of a boy who was homozygous for normal allele. The healthy child was born.

\section{Discussion}

Since the first introduction of PGD, which helps women to experience a healthy pregnancy, the number of PGD cycles performed worldwide has increased dramatically (13).

Here we report the first design and setup of PGD test for a family with steroid-resistant nephrotic syndrome using both direct mutation analysis by cycle sequencing and indirect analysis by flanking STR markers.

Based on the STR markers on both sides of the NPHS2 gene the embryos 1 and 5 were heterozygous. However Sanger sequencing showed only one mutant allele. This was most likely due to the allele dropout in amplification of NPHS2 exon 1 for cycle sequencing.

Embryo 2 was revealed to be homozygous for mutant allele.

Both sequencing and linkage analysis showed that the embryo 4 had at least one normal allele. Regarding the second allele, STR analysis revealed the 239bp allele for downstream marker D1S3760 which is linked to normal allele 


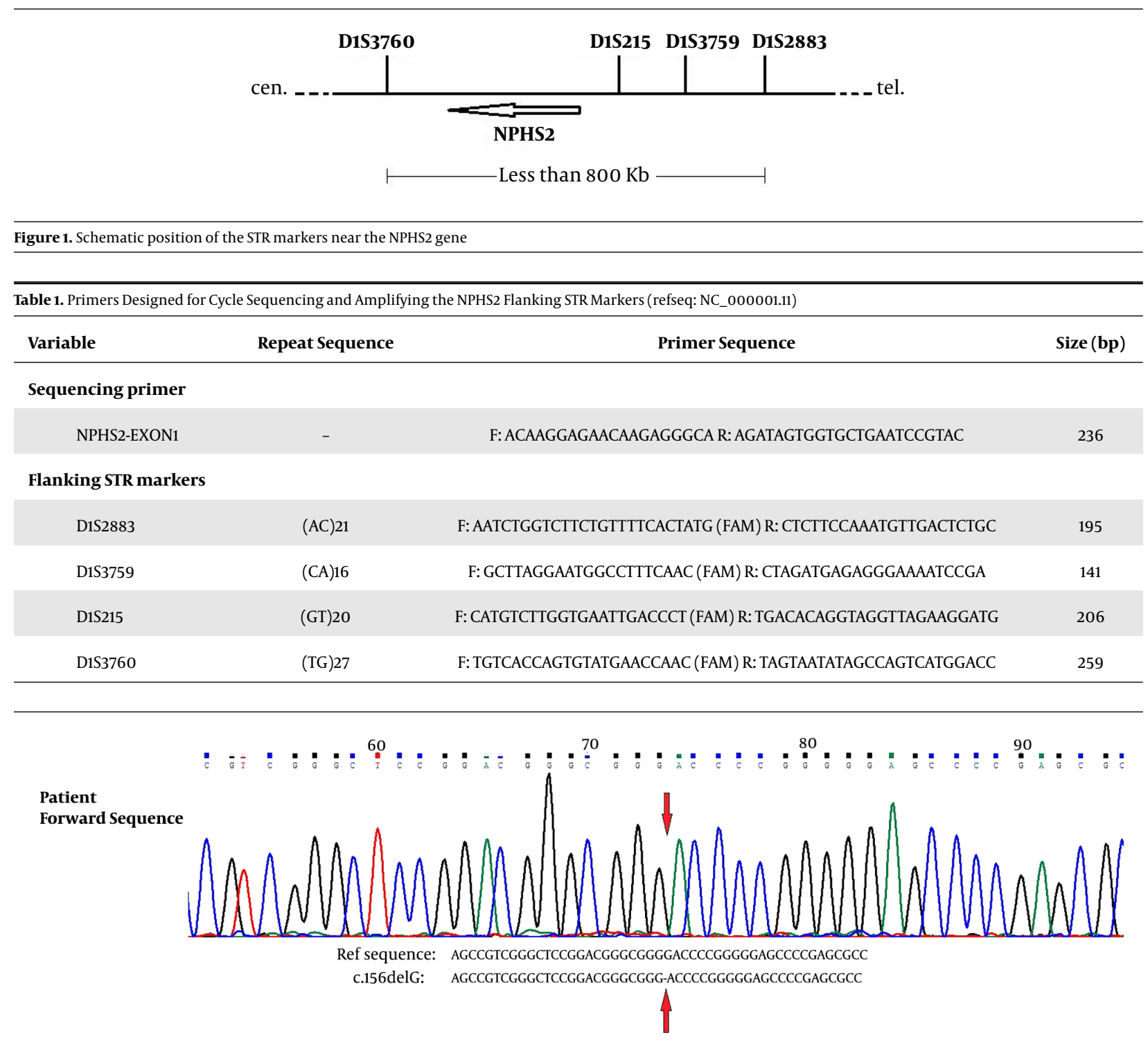

Figure 2. Sequencing result of the NPHS2 exon 1 of the affected son in comparison to the reference sequence.

and the 210bp allele for upstream marker D1S215 which is linked to mutant allele. This might have happened due to a recombination. Since allele dropout in PCR for cycle sequencing was probable too, we couldn't determine the status of the second NPHS2 allele based on these evidences. Two other upstream markers had allele dropout.

Embryo 4 was transferred and resulted in an unaffected pregnancy and birth of one healthy boy.

In this PGD test, allele dropout was observed in D1S2883, D1S3759 and NPHS2 exon 1. D1S215 and D1S3760 markers didn't show ADO.
In conclusion, PGD can be carried out as an accurate option for couples who are carrier for mutations in NPHS2 gene in order to screen for a healthy embryo before implantation.

According to the ESHRE guideline analysis of at least two flanking markers which are closely linked to the causative gene in indirect mutation PCR is recommended and reduces the risk of $\mathrm{ADO}$ and transferring of an affected embryo from $5 \%$ to $1 \%$. However while using only one marker at each side of the mutated gene, if amplification of one marker fails there will be no diagnosis for that embryo 

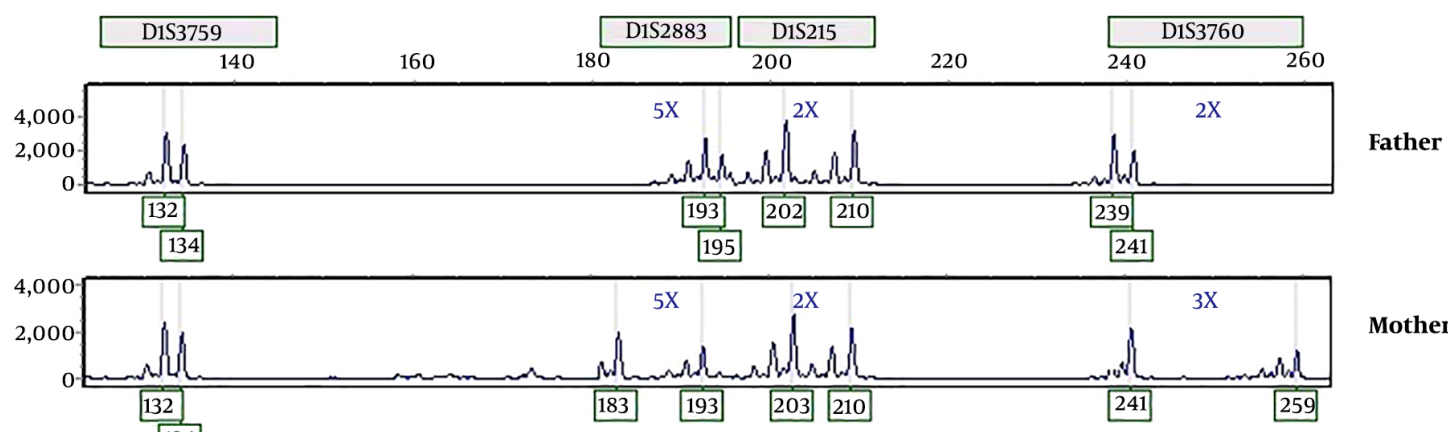

Mother

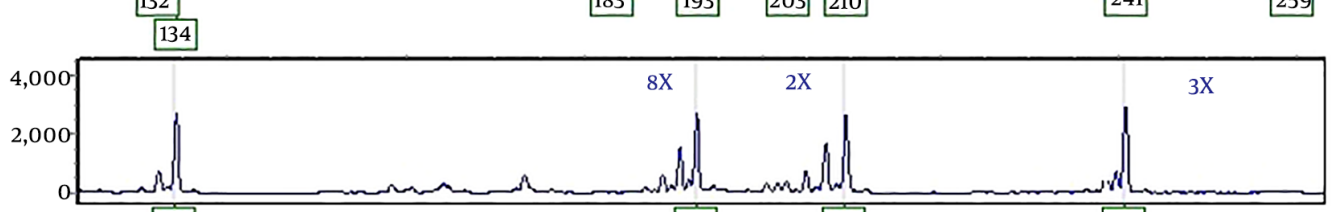

fected

son

$\begin{array}{llll}134 & 193 & 210 & 241\end{array}$

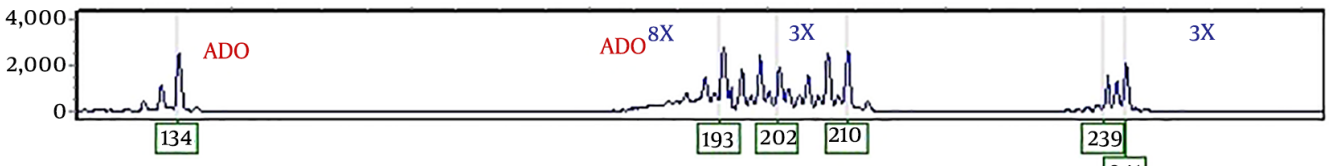

241

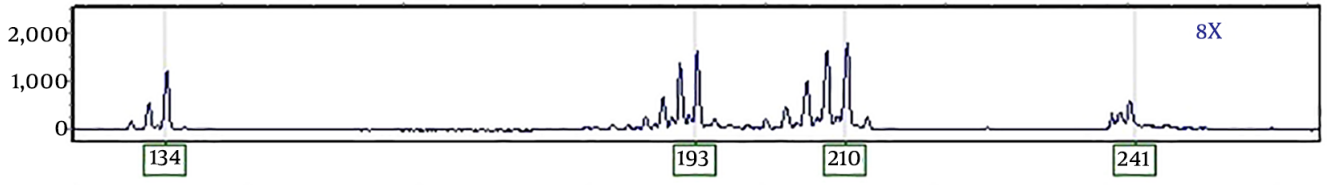

Embryo 2

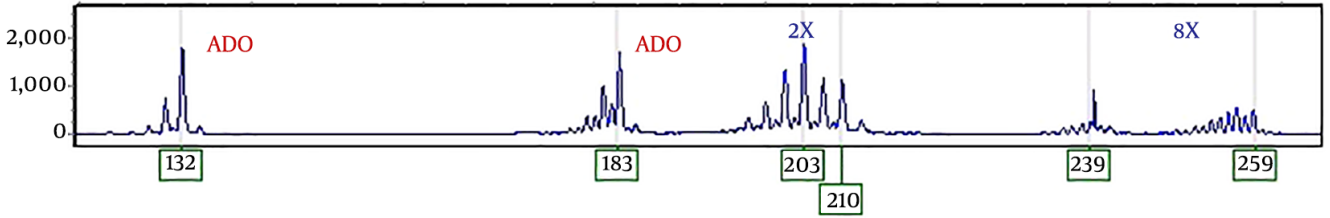

Embryo 1

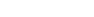

Embryo 4

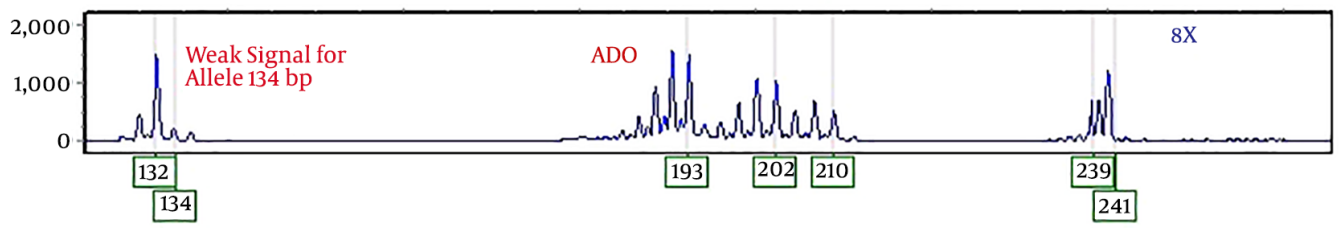

Embryo 5

Figure 3. Electropherogram of the STRs linked to NPHS2 gene in PGD test. (ADO: allele drop out)

(14). Therefore we recommend increasing the number of the STR markers to two at the downstream of the NPHS2 gene especially in cases that direct mutation analysis such as cycle sequencing is not applied.

\section{Acknowledgments}

The authors thank the personnel of Tehran Medical Genetics Laboratory for their help and support. This project was financially supported by Tehran Medical Genetics Labora $\neg$ tory.

\section{Footnotes}

Authors' Contribution: Faravareh Khordadpour Deilamani : Designed the test/ setup and performed the pre-PGD and PGD tests/analyzed and interpreted the data/ wrote the draft of the article/ approved the final version of the manuscript. 


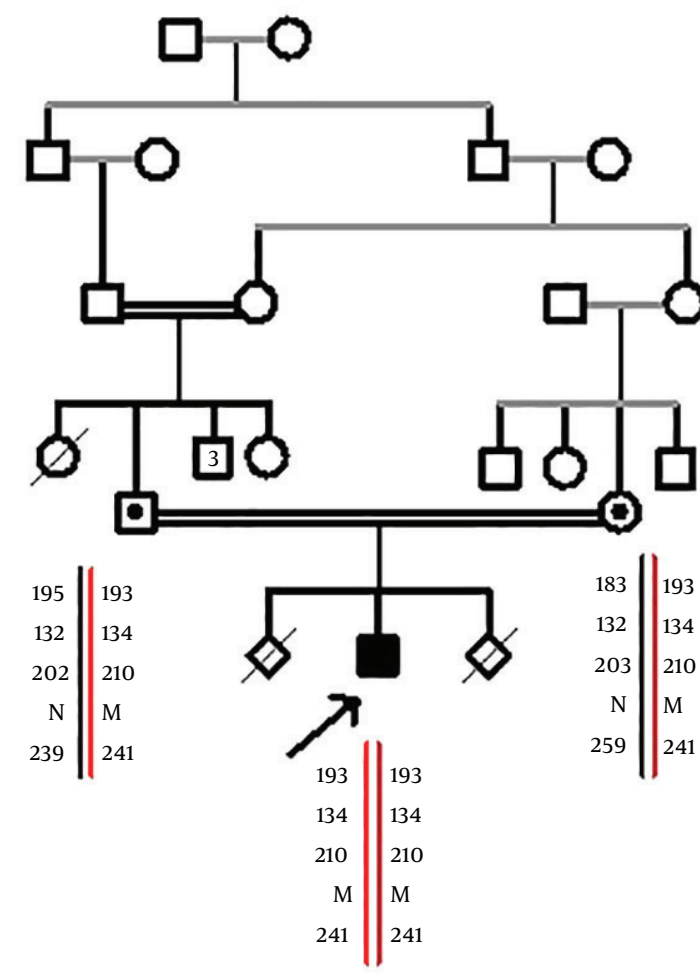

S TR Order

D1S2883

D1S3759

D1S215

NPHS2

D1S3760

\begin{tabular}{|c|c|c|c|c|c|c|c|c|}
\hline & \multicolumn{2}{|c|}{ Embryno 1} & \multicolumn{2}{|c|}{ Embryno 2} & \multicolumn{2}{|c|}{ Embryno 4} & \multicolumn{2}{|c|}{ Embryno 5} \\
\hline & ADO & 193 & 193 & 193 & 183 & $\mathrm{ADO}$ & $\mathrm{ADO}$ & 193 \\
\hline \multirow{4}{*}{ S TR Marker Results } & $\mathrm{ADO}$ & 134 & 134 & 134 & 132 & $\mathrm{ADO}$ & 132 & 134 \\
\hline & 202 & 210 & 210 & 210 & 203 & 210 & 202 & 210 \\
\hline & $\mathrm{N}$ & M & M & M & $\mathrm{N}$ & $?$ & $\mathrm{~N}$ & M \\
\hline & 239 & 241 & 241 & 241 & 259 & 239 & 239 & 241 \\
\hline Sequencing Results: & \multicolumn{2}{|c|}{ Mutant Allele } & \multicolumn{2}{|c|}{ No Result } & \multicolumn{2}{|c|}{ Nonmal Allele } & \multicolumn{2}{|c|}{ Mutant Allele } \\
\hline
\end{tabular}

Figure 4. Results of STR-based linkage analysis and cycle sequencing used in PGD for steroid-resistant Nephrotic syndrome. The DNA from blastomeres of embryos 3 and 6 were not amplified by whole genome amplification, and could not be processed in later stages. (ADO: allele drop out)

Conflict of Interests: None declared.

Ethical Approval: The study was approved by the local ethics committee.

Funding/Support: This project was financially supported by the Tehran Medical Genetics Laboratory.

Informed Consent: the informed consent was obtained from the parents.

\section{References}

1. Boute N, Gribouval O, Roselli S, Benessy F, Lee H, Fuchshuber A, et al.
NPHS2, encoding the glomerular protein podocin, is mutated in autosomal recessive steroid-resistant nephrotic syndrome. Nat Genet. 2000;24(4):349-54. doi: 10.1038/74166. [PubMed:10742096].

2. Weber S, Gribouval O, Esquivel EL, Moriniere V, Tete MJ, Legendre C, et al. NPHS2 mutation analysis shows genetic heterogeneity of steroid-resistant nephrotic syndrome and low posttransplant recurrence. Kidney Int. 2004;66(2):571-9. doi: 10.1111/j.15231755.2004.00776.x. [PubMed: 15253708].

3. Santin S, Tazon-Vega B, Silva I, Cobo MA, Gimenez I, Ruiz P, et al. Clinical value of NPHS2 analysis in early- and adult-onset steroidresistant nephrotic syndrome. Clin J Am Soc Nephrol. 2011;6(2):344- 
54. doi: 10.2215/CJN.03770410. [PubMed: 20947785]. [PubMed Central: PMC3052225].

4. Handyside AH, Kontogianni EH, Hardy K, Winston RM. Pregnancies from biopsied human preimplantation embryos sexed by Y-specific DNA amplification. Nature. 1990;344(6268):768-70. doi: 10.1038/344768a0. [PubMed: 2330030].

5. Verlinsky Y, Ginsberg N, Lifchez A, Valle J, Moise J, Strom CM. Analysis of the first polar body: preconception genetic diagnosis. Hum Reprod. 1990;5(7):826-9. doi: 10.1093/oxfordjournals.humrep.a137192. [PubMed: 2266156].

6. Handyside AH, Lesko JG, Tarin JJ, Winston RM, Hughes MR. Birth of a normal girl after in vitro fertilization and preimplantation diagnostic testing for cystic fibrosis. N Engl J Med. 1992;327(13):905-9. doi: 10.1056/NEJM199209243271301. [PubMed: 1381054].

7. Hussey ND, Davis T, Hall JR, Barry MF, Draper R, Norman RJ, et al. Preimplantation genetic diagnosis for beta-thalassaemia using sequencing of single cell PCR products to detect mutations and polymorphic loci. Mol Hum Reprod. 2002;8(12):1136-43. doi: 10.1093/molehr/8.12.1136. [PubMed: 12468648].

8. Satirapod C, Sukprasert M, Panthan B, Charoenyingwattana A, Chitayanan P, Chantratita W, et al. Clinical utility of combined preimplantation genetic testing methods in couples at risk of passing on beta thalassemia/hemoglobin E disease: A retrospective review from a single center. PLoS One. 2019;14(11). e0225457. doi: 10.1371/journal.pone.0225457. [PubMed: 31751397]. [PubMed Central: PMC6872132].

9. DeUgarte C, Ambartsumyan G, Li M, Verlinsky Y, Hill D, DeCherney A. Successful Selection of Normal Embryos Using PGD in a Woman with Spinocerebellar Ataxia Type 3. Fertility and Sterility. 2005;83(5):S30-1. doi:10.1016/j.fertnstert.2005.01.077.

10. Rechitsky S, Verlinsky O, Kuliev A, Ozen S, Laziuk K, Beck R, et al. Preimplantation genetic diagnosis for early-onset torsion dystonia. Reproductive BioMedicine Online. 2004;8(2):224-8. doi: 10.1016/s14726483(10)60520-2.

11. Merker VL, Murphy TP, Hughes JB, Muzikansky A, Hughes MR, Souter I, et al. Outcomes of preimplantation genetic diagnosis in neurofibromatosis type 1. Fertil Steril. 2015;103(3):761-8 e1. doi: 10.1016/j.fertnstert.2014.11.021. [PubMed: 25557241].

12. Sermon K, Lissens W, Joris H, Seneca S, Desmyttere S, Devroey P, et al Clinical application of preimplantation diagnosis for myotonic dystrophy. Prenatal Diagnosis: Published in Affiliation With the International Society for Prenatal Diagnosis. 1997;17(10):925-32.

13. Verlinsky Y, Rechitsky S, Verlinsky O, Strom C, Kuliev A. Preimplantation testing for phenylketonuria. Fertility and Sterility. 2001;76(2):3469. doi:10.1016/s0015-0282(01)01912-4.

14. Harton GL, De Rycke M, Fiorentino F, Moutou C, SenGupta S, TraegerSynodinos J, et al. ESHRE PGD consortium best practice guidelines for amplification-based PGD. Human reproduction. 2011;26(1):33-40. 